African J. Biol. Sci., 15 (1): 219-234 (2019)

ISSN 1687-4870

www.ajbs.journals.ekb.eg

e- ISSN 2314-5501 (online)

e.mail: aasdjournal@yahoo.com

\title{
Assessment of genetic diversity among some fennel cultivars (Foeniculum vulgare Mill.) by ISSR and SCoT Markers
}

\author{
Walaa A. Ramadan, R.M. Shoaib, Rania T. Ali* and N.S. Abdel-Samea \\ Genetics and cytology Department, Genetic Engineering and Biotechnology Division, National \\ Research Centre. 33 El Buhouth st., Dokki, Cairo, Egypt. \\ * Correspondence Author E-mail: raniatawfick@hotmail.com
}

\begin{abstract}
Fennel (Family Apiaceeae) is one of those strategic medicinal and aromatic plants in Egypt with great value as an economical exported plant. Fennel undergoes propagation through breeding of some fennel cultivars from different world region in order to keep out of genetic deterioration in the native Egyptian cultivars, and enrich its favorable active ingredients. The advanced plant breeding programs depend on some markers such as morphological, cytological, biochemical and molecular markers to study its genetic diversity.

In this work two molecular markers ISSR and SCoT markers were used to assessment of genetic diversity among six fennel cultivars (Foeniculum vulgare Mill.) planted in Egypt; three of Egyptian origin (Balady, Elalamy and Azoricum) and the other ones are of different origin (Holland, Indian and German). The analyzed data reveals that the ISSR marker is more discriminating, provides more informative data than SCoT marker and can be reliable to evaluate the genetic diversity among the six examined cultivars as it performs the highest percentage of polymorphism 61\% and higher unique bands number (4). According to ISSR molecular marker German and Indian cultivars are close distant; can exchange gene in between them smoothly despite the ultimate change in the environmental and weather conditions of their origin land.

In conclusion, according to Nie's and li's coefficient based on SCoT marker analysis between the cultivars; Indian cultivar was quite distant from Azoricum by the same time that Azoricum is close distant to Holland, as well as; Elalamy is close distant to Balady.
\end{abstract}

Key words: Genetic diversity, Fueniculum vulgare, molecular markers, SCoT, ISSR.

\section{INTRODUCTION}

Fennel (Foeniculum vulgare Mill.), commonly named: Shammor, Shammar, Pespas, Bitter fennel, Sweet fennel and Moroccan Crab, is an important multi purposes plant. Native in south Europe and Asia minor, cultivated in USA, UK and moderate Eurasia. It is a perennial herb with yellow flowers and belonging to family Apiaceeae. Suitable for planting in wide range of well drained soil from black clay soil to yellow sand soil. It gains its importance from high medicinal uses as it contains: Anethol which is an anti cancer compound fight the liver and breast cancer, Quercetin which is anti-inflammation compound, dietary nitrates which lower the blood pressure and it also contain melatonin which help in lowering the excess body weight. Also fennel contains $\mathrm{A}$ and $\mathrm{C}$ vitamins and rich in fibers (Wesam et al., 2015). In Indian folk, dry seeds may be eaten to improve vision and treat glaucoma, in Egyptian folk; boiling fennel in water may be the first baby diet as it relief its digestive system; later on fennel water 
Walaa A. Ramadan et al.

becomes one of the constitutes the domestic 'gripe water', used to correct flatulence of infants (Rather et al., 2012). Fennel essential oil is as value as gold for it has antibacterial, antifungal, antioxidant and anticancer activity (Lucinewton et al., 2005 \& Wesam et al., 2015).

In Egypt, the medicinal and aromatic plants such as cinnamon, lemon grass, fennel, Anis....etc. represent about $0.8 \%$ from total cultivated plant, $90 \%$ from the local production is exported to the international markets and considered as an important source of national income. Fennel is one of those strategic medicinal plants, represented in common fennel cultivars in Egypt such as:

1. German fennel which belongs to the sweet fennel with anethole ingredient $70-80 \%$ of total aromatic oil.

2. Indian cultivar which belongs to the bitter fennel with anethole is $\sim 70 \%$ of aromatic oil.

3. Balady (local cultivar) belongs to bitter fennel with anethole is $~ 5-10 \%$ of oil.

4. Holland cultivar belongs to bitter fennel which gives the highest value of anethole $\sim 70-80 \%$ of total oil.

5. Azoricum cultivar.

6. Elalamy cultivar.

Egyptian fennel ecotype (Balady) lately suffered from deterioration in its volatile oil to $1.7-2 \%$ also its Anethol ingredient lowered by $40-50 \%$ which in turn lower its exportation and national income as now it retarded to the sixth place instead of the second place. This led the Egyptian agriculture ministry to breed some other fennel cultivars in order to improve the fennel production for its high economic importance. Project (EMAP) for "the medicinal and aromatic plants evolution for production" succeeded in breeding the fennel "Holland" cultivar in Egypt as it produce about $80-90 \%$ of (Balady) cultivar production with volatile oil $2.5-3 \%$ and
Athenol 75-80\%. It is well known that, the success of any breeding program depends on the sufficient diversity available to allow the production of new varieties with improved crop productivity and tolerance to biotic and abiotic stress. Variation in plant genetic pool gives the plant breeders a valuable opportunity to develop new and improved cultivars with much more desirable characteristics. This inspires the scientists to study the genetic diversity in different fennel cultivars to understand of their genetic relationships which could improve fennel conservation and utilization and lead to new cultivar development. Better understanding of genetic diversity will help in determining what to plant as well as where to plant. Genetic diversity of crop plants is the foundation for the sustainable development of new varieties.

In recent years, DNA-based molecular markers have been used for assessment of the genetic diversity between germplasm in many plant species. Molecular markers have shown advantages over other markers because of their neutrality and feasibility, they do not depend on age and tissue type and also are not influenced by the environmental conditions. (Zietkiewicz et al., 1994) stated that inter simple sequence repeat (ISSR) markers detect the polymorphism in the non coding region have been successfully used to identify and determine relationships at the species. ISSR markers are also used to identify and discriminate polymorphism among population and varieties in many aromatic and medicinal plants (Sangwan et al., 1999; Nan et al., 2003). ISSR markers have been efficiently used for study of genetic diversity of different medicinal plant species and crops such as Phaseolus vulgaris L. (Galvan et al., 2003), barley (Yong-Cui et al, 2005), Artemisia capillaries (Shafie et al, 2009), Lippia alba Mill. (Manica-Cattani et al, 2009) and Achillea millefolium (Farajpour 
et al, 2012). This method is widely applicable because it is rapid, inexpensive, require small amounts of template DNA. A newly developed DNA marker technique start codon targeted (SCoT) polymorphic markers were used to assess genetic relationships among varieties. As it is simple, highly reproducible due to the use of longer primers, these kinds of markers mostly correlated to functional gene and their corresponding traits or to the gene immediate flanking sequences. Yaday and Malik (2016) found that the SCoT marker can be very effective for characterization of genetic diversity in medicinal plant like
Foeniculum vulgare Mill. In this work the ISSR and SCoT markers were used to assessment six fennel different cultivars coming from different home origin to determine there genetic diversity which in turn influence the genetic exchange between them.

\section{MATERIALS AND METHODS 1-Plant materials:}

Six fennel cultivars (Foeniculum vulgare Mill) cultivated in Egypt; three cultivars are native in Egypt while the other three were coming from different origins, were examined in this work for the study of their molecular diversity (Table 1).

Table 1: Names and origin land of the six fennel cultivars (Foeniculum vulgare Mill).

\begin{tabular}{|l|l|}
\hline \multicolumn{1}{|c|}{ Cultivar Name } & \multicolumn{1}{c|}{ Origin } \\
\hline Holland & Holland \\
\hline Azoricum & Egypt \\
\hline Indian & India \\
\hline German & Germany \\
\hline Balady & Egypt \\
\hline El alamy & Egypt \\
\hline
\end{tabular}

\section{2- Methods:}

\section{2.a- DNA extraction and purification:}

Total genomic DNA was extracted from young and fresh leaves of Foeniculum vulgare Mill. as described by Dellaporta et al. (1983).

\section{2.b- ISSR and SCoT analysis:}

To achieve ISSR and SCoT fragments, first the selection of recommended primers was performed six ISSR primers and eight SCoT and. (Tables 2 $\& 3$ ) lists the base sequences of these DNA primers. PCR technique was performed in $25 \mu \mathrm{l}$ volume containing: master mix beads, $10 \mu \mathrm{l}$ buffer $(10 \mathrm{X}), 1 \mu \mathrm{l}$ primer (100 pmol), $1 \mu \mathrm{l}$ DNA template (50 ng) and $13 \mu \mathrm{H} \mathrm{O}$ sterile. The amplification was carried out in cycler programmed as follows: 1 cycle of $94^{\circ} \mathrm{C} / 2$ min, 35 cycles of $\left(94^{\circ} \mathrm{C} / 1 \mathrm{~min}\right.$, $48^{\circ} \mathrm{C} / 2 \mathrm{~min}$ and $\left.72^{\circ} \mathrm{C} / 2 \mathrm{~min}\right), 1$ cycles of $72^{\circ} \mathrm{C} / 7 \mathrm{~min}$. The primer annealing degrees were varied according to the melting point of each primer. Agarose gel was used for separating the PCR products of amplified DNA fragments by electrophoresis. The agaros gel was prepared by dissolving $1.2 \mathrm{~g}$ agarose in $100 \mathrm{ml}$ buffer including $40 \mathrm{mM}$ Tris acetate and $2 \mathrm{mM}$ Na EDTA.2H O. The gel was stained with ethidium bromide, photographed under UV light, scanned using a Gel-Documentation system and the data were analyzed using Bio-Rad Model 620 Software Programs, USA. Genetic similarity was estimated using Nei-Li's similarity index Nei and Li (1979). A dendrogram was constructed on the basis of the similarity matrix data by un-weighted pair group with arithmetic average (UPGMA) cluster analysis using the software MEGA program. 
Walaa A. Ramadan et al.

Table (2): Six ISSR primers were used to characterize six fennel cultivars with the following sequences:

\begin{tabular}{|l|c|}
\hline \multicolumn{1}{|c|}{ Primer names } & Sequences $5^{\prime} \rightarrow 3^{\prime}$ \\
\hline 49A & CAC ACA CAC ACA AG \\
\hline 44B & CTC TCT CTC TCT CTC TGC \\
\hline HB-9 & GTG TGT GTG TGT GG \\
\hline HB-11 & GTG TGT GTG TGTCC \\
\hline HB-12 & CAC CAC CAC GC \\
\hline HB-15 & GTG GTG GTG GC \\
\hline
\end{tabular}

Table (3): Eight SCoT primers were used to characterize six fennel cultivars with the following sequences:

\begin{tabular}{|c|c|}
\hline Primer names & $\begin{array}{c}\text { Sequences } \\
5^{\prime} \rightarrow 3^{\prime}\end{array}$ \\
\hline SCoT 1 & ACG ACA TGG CGA CCA CGC \\
\hline SCoT 2 & ACC ATG GCT ACC ACC GGC \\
\hline SCoT 3 & ACG ACA TGG CGA CCC ACA \\
\hline SCoT 6 & CAA TGG CTA CCA CTA CAG \\
\hline SCoT 8 & ACA ATG GCT ACC ACT GAG \\
\hline SCoT 10 & ACA ATG CTA CCA CCA AGC \\
\hline SCoT 11 & ACA ATG GCT ACC ACT ACC \\
\hline SCoT 12 & CAA CAA TGG CTA CCA CCG \\
\hline
\end{tabular}

\section{RESULTS}

\section{1-ISSR Analysis:}

The generated monomorphic/ polymorphic bands, unique bands, the percentage and the average of polymorphism in 6 cultivars are presented in Table (4) and figure (1).

In the present study, six selected ISSR primers were used to examine the genetic polymorphism among the six concerned cultivars of Foeniculum vulgare plants. The obtained data were illustrated in Table (4) and Fig. (1) Clarify that; the size of the amplified bands ranged from about 155 bp. (after primer HB-12) to 2000 bp. (after primer 44B). Number of bands ranged from 12 bands (primer 44B) to 4 bands (after primers HB-9 and HB-15) with an average of 6.33 amplicons per primer. All of the 6 primers detected polymorphic patterns except primer HB-9 and revealed a total number of 38 amplicons with $61 \%$ level of polymorphism. The highest percentage of polymorphism was $100 \%$ (after primer 49A) and the lowest percentage of polymorphism was $0.00 \%$ (after primer HB-9).The number of monomorphic amplicons varied from 0 (primer 49A) to 4 (after primers HB-9 and HB-15) in average of 2.5 monomorphic band per primer. However, the number of polymorphic bands varied from 0 (primer HB-9) to 8 (primer 44B) in average of 3.83 polymorphic amplicons per primer. Moreover, the number of unique fragments varied from 0 (49A and44B) to 2 (HB-9, HB-11, HB-12 and HB-15) with an average of $\mathbf{0 . 6 6}$ unique fragments per primer. 
Assessment of genetic diversity among some fennel cultivars (Foeniculum vulgare Mill.) by ISSR and SCoT Markers

Table (4): Primers name, total bands number, monomorphic, polymorphic \& unique bands and percentage of polymorphism as revealed by 6 ISSR primers in between the six fennel cultivars Foeniculum Vulgare Mill.

\begin{tabular}{|l|c|c|c|c|c|}
\hline \multicolumn{1}{|c|}{ Primer name } & T B & M B & P B & U B & P \% \\
\hline 49A & 6 & 0 & 6 & 2 & 100 \\
\hline 44B & 12 & 4 & 8 & 2 & 66.66 \\
\hline HB-9 & 4 & 4 & 0 & - & 0.00 \\
\hline HB-11 & 7 & 2 & 5 & - & 71.4 \\
\hline HB-12 & 5 & 3 & 2 & - & 40.0 \\
\hline HB-15 & 4 & 2 & 2 & - & 50.0 \\
\hline Total & 38 & 15 & 23 & 4 & - \\
\hline average & 6.3 & 2.5 & 3.8 & - & 60.3 \\
\hline
\end{tabular}

TB (Total bands), MB (Monomorphic bands), PB (Polymorphic bands), UB( Unique bands) and $\mathrm{P} \%$ (percentage of polymorphism).

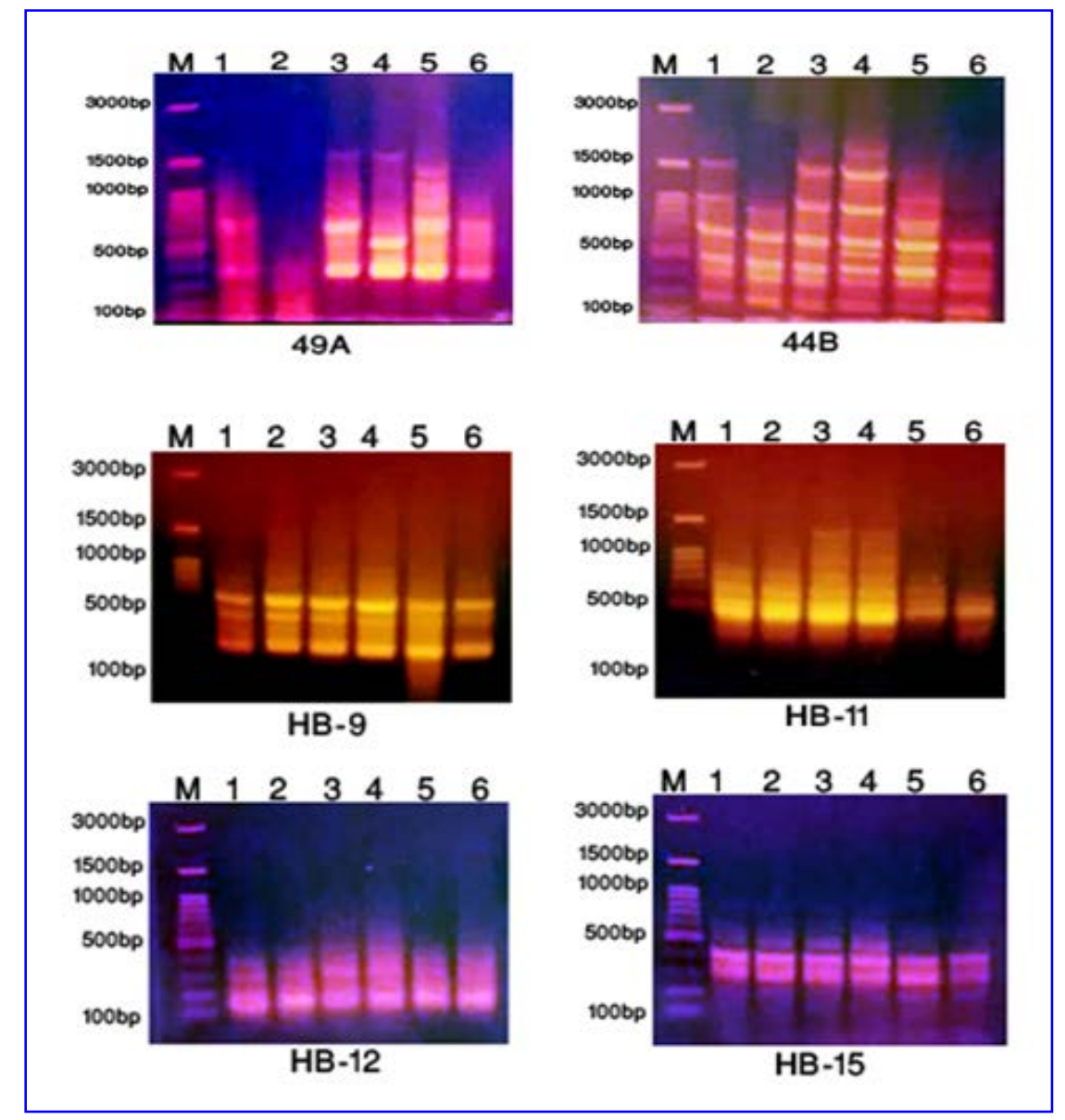

Fig. (1): ISSR banding patterns of the six fennel cultivars (Foeniculum Vulgare Mill.) with six arbitrary primers. M: marker, 1: Holland , 2: Azoricum, 3: Indian, 4:

German, 5: Balady and 6: Elalamy 
Walaa A. Ramadan et al.

A highest polymorphism $100 \%$ was obtained by primer 49A, with zero \% monomorphic fragments; six polymorphic bands. Two positive markers (unique bands) with molecular sizes 1375 and 560 bp were identified only with Balady and German respectively. Whereas the fragment with molecular size 260 bp. was detected with all cultivars except cultivars Azoricum only so, it was considered as a negative marker for Azoricum. The fragments with molecular sizes 845 and 730 bp. were appeared with all cultivars and absent with cultivars Holland, Azoricum and German respectively. Consversely, 1845 bp. sized fragment was only showed with cultivars Indian and German. Two positive markers were distinguished by primer 44B with molecular sizes 2000 and 640 bp were observed with German and Balady, respectively. Thus eight polymorphic fragments and four monomorphic fragments were recognized, $66.66 \%$ polymorphism. The fragments with molecular sizes 570 and 465 bp were exhibited with cultivars German and Balady only. The fragments with molecular sizes 1100, 1060 and 760 bp were appeared with two cultivars only; Holland, Balady - Indian, German and Azoricum, Balady, respectively. The fragment with molecular size 1630 bp. was scored with cultivars Holland, Indian and German. No polymorphism has recorded by primer HB9. Four monomorphic bands were identified with molecular sizes 625, 500, 430 and 235 (all cultivars). The primer HB-11 was scored 2 monomorphic bands and 5 polymorphic, $71.4 \%$ polymorphism.

However, the fragments at molecular sizes, 1285 and 1000 bp. were distinguished with cultivars Indian and German only and absent with all cultivars. On the contrary, the fragments with molecular sizes 825 and
480 bp. were showed with most cultivars except the German and Balady. Polymorphism was $40 \%$ and only one negative marker was exhibited by primer HB-12, this fragment at molecular size 475 bp. was appeared with all cultivars and absent with Holland only. Three monomorphic bands and two polymorphic fragments were distinguished. At the same time, the fragment at molecular size $630 \mathrm{bp}$. was recognized with cultivars Indian and German only. Polymorphism was 50\% with primer HB-15, two monomorphic and two polymorphic fragments were distinguished. The fragment at molecular size $515 \mathrm{bp}$. was observed with most of the cultivars except the German and Balady. On contrast, the fragment at molecular size 300 bp. was recognized only with cultivars German and Balady. The results of ISSR analysis were translated to generate the dendrogram and similarity index (Fig. 2 \& Table 5)

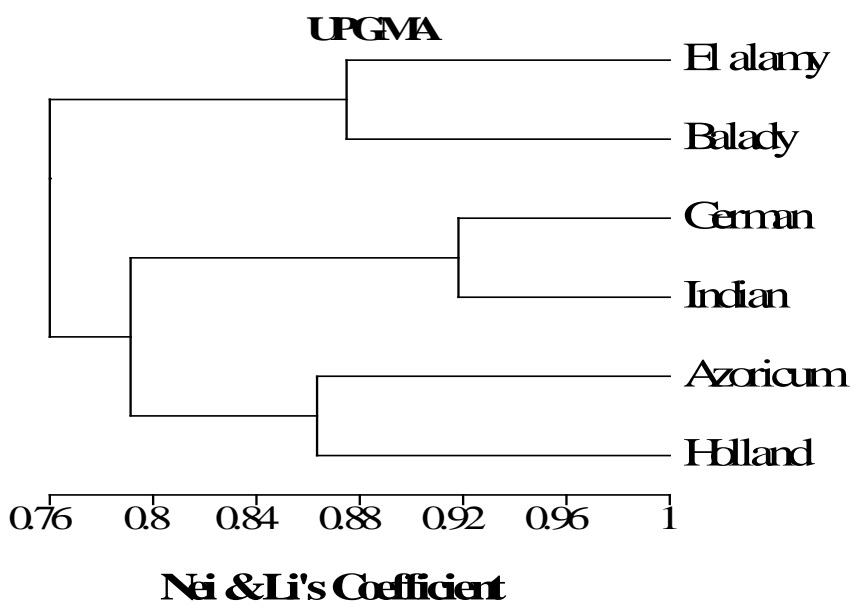

Fig. (2): Dendrogram represents the genetic relationships among the six fennel cultivars using UPGMA cluster analysis generated from of ISSR marker. 
Assessment of genetic diversity among some fennel cultivars (Foeniculum vulgare Mill.) by ISSR and SCoT Markers

Table (5): Similarity Index Using ISSR analysis for the six fennel cultivars (Foeniculum Vulgare Mill.).

\begin{tabular}{|c|c|c|c|c|c|c|}
\hline Cultivars & Holland & Azoricum & Indian & German & Balady & El alamy \\
\hline Holland & $\mathbf{1}$ & & & & & \\
\hline Azoricum & $\mathbf{0 . 8 6}$ & 1 & & & & \\
\hline Indian & $\mathbf{0 . 8 5}$ & $\mathbf{0 . 8}$ & 1 & & & \\
\hline German & $\mathbf{0 . 7 6}$ & $\mathbf{0 . 7 5}$ & $\mathbf{0 . 9 2}$ & 1 & & \\
\hline Balady & $\mathbf{0 . 7 6}$ & 0.75 & 0.71 & 0.71 & 1 & \\
\hline El alamy & $\mathbf{0 . 8 2}$ & 0.81 & 0.72 & 0.72 & 0.875 & 1 \\
\hline
\end{tabular}

\section{2- SCoT analysis:}

The generated DNA monomorphic /polymorphic bands, unique bands and the percentage \& average of polymorphism in six cultivars are presented in Table (6) and Figure (2).

Table (6): Primers name, Total bands number, Monomorphic, polymorphic \& unique bands and percentage of polymorphism as revealed by 8 SCoT primers among the six fennel cultivars Foeniculum Vulgare Mill.

\begin{tabular}{|c|c|c|c|c|c|}
\hline Primer & TB & MB & Pb & UB & P \% \\
\hline SCoT 1 & 9 & 5 & 4 & - & 44.4 \\
\hline SCoT 2 & 7 & 2 & 5 & - & 71.5 \\
\hline SCoT 3 & 7 & 4 & 3 & 1 & 43 \\
\hline SCoT 6 & 7 & 1 & 6 & - & 85.7 \\
\hline SCoT 8 & 4 & 2 & 3 & 1 & 75 \\
\hline SCoT 10 & 9 & 3 & 6 & 1 & 66.6 \\
\hline SCoT 11 & 10 & 7 & 3 & - & 30 \\
\hline SCoT 12 & 6 & 2 & 4 & - & 80 \\
\hline Total & 59 & 26 & 34 & 3 & \\
\hline average & 7.38 & 3.25 & 4.25 & & 57.6 \\
\hline
\end{tabular}

TB (Total bands), MB (Monomorphic bands), PB (Polymorphic bands), UB (Unique bands) and $\mathrm{P} \%$ (percentage of polymorphism). 
Walaa A. Ramadan et al.
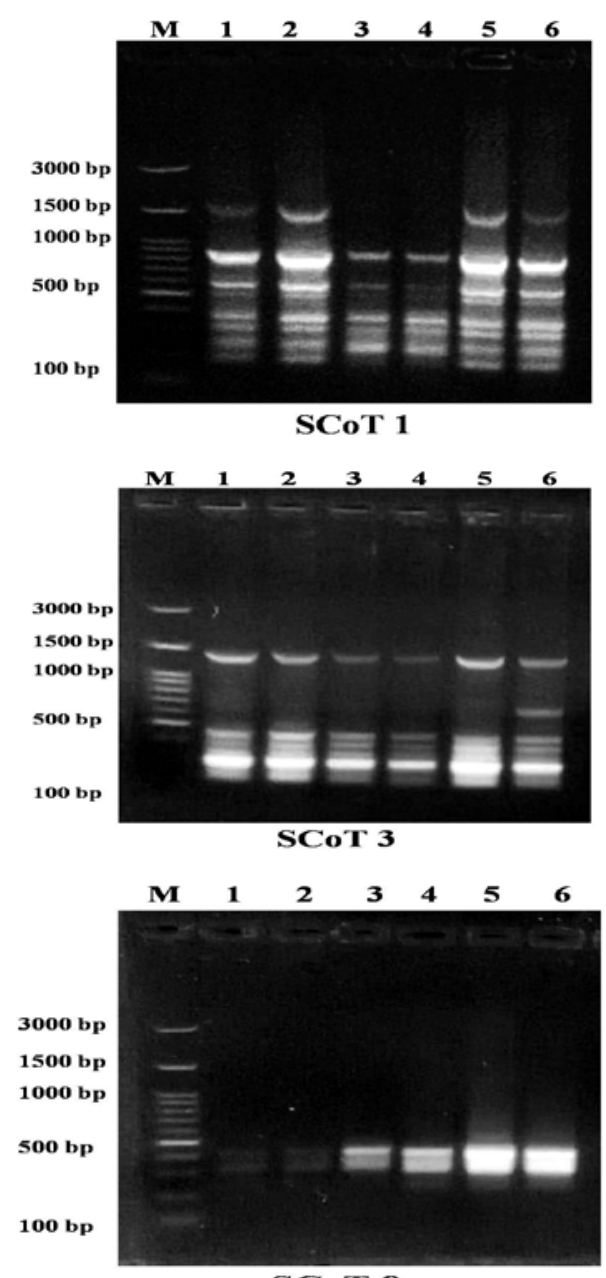

SCoT 8

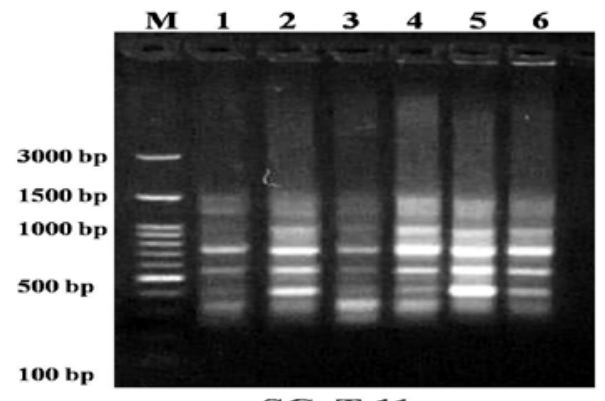

SCoT 11

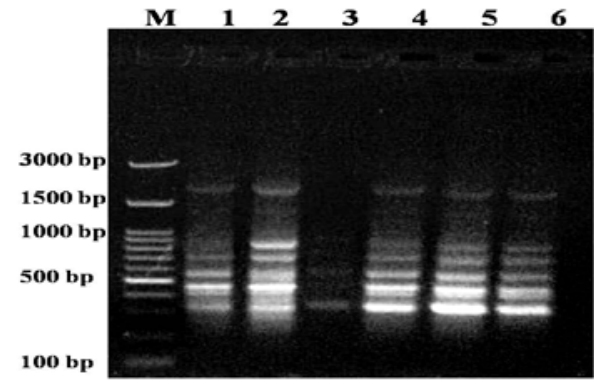

SCoT 2

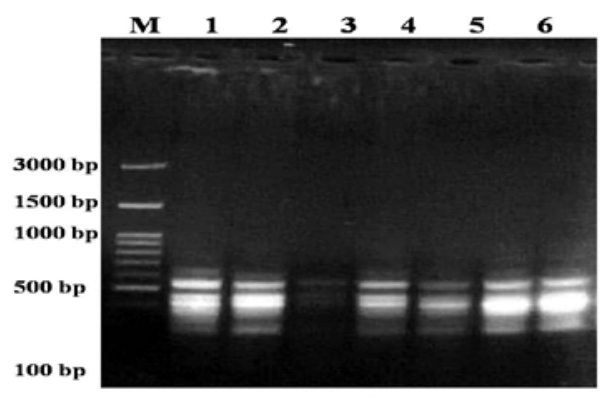

SCoT 6
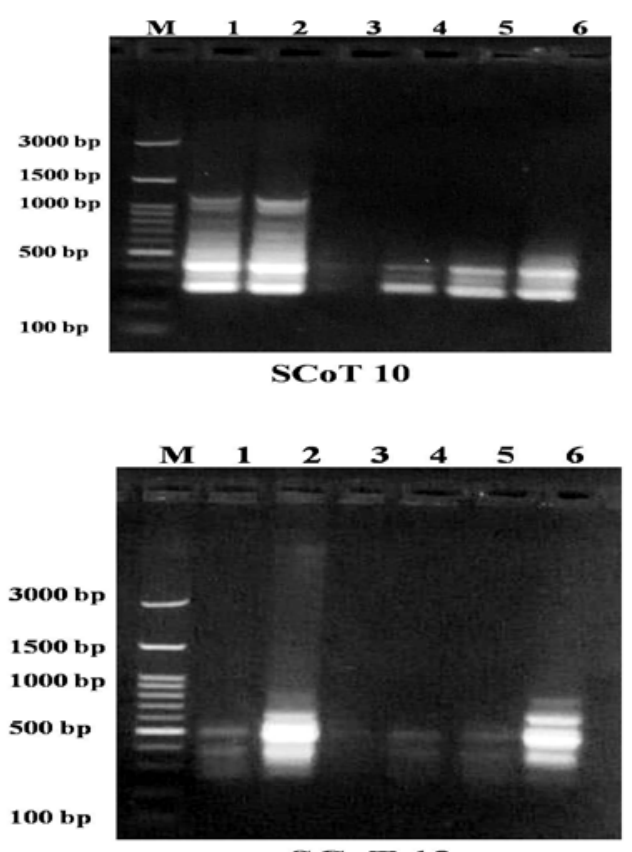

SCoT 12

Fig. 3: SCoT banding patterns of the six fennel cultivars with 8 arbitrary primers. M: marker, 1: Holland , 2: Azoricum, 3: Indian, 4: German, 5: Balady and 6: Elalamy.

In the present investigation, eight SCoT primers were used to investigate the genetic polymorphism among the six selcted cultivars of $F$. vulgare plants. As shown in Table (6) and Figure (3), the size of the amplified bands ranged from about
175 bp. (after primer SCoT 1) to 2110 (after primer SCoT 2). Number of bands ranged from 10 bands (primer SCoT 11) to 4 bands (after primer SCoT 8) with an average of 7.37 amplicons per primer. All of the 8 primers detected polymorphic patterns and 


\section{Assessment of genetic diversity among some fennel cultivars (Foeniculum vulgare Mill.) by ISSR and SCoT Markers}

revealed a total number of 59 amplicons with $57.62 \%$ level of polymorphism. The highest percentage of polymorphism was 85.7\% (after primer SCot 6) and the lowest percentage of polymorphism was $30.0 \%$ (after primer SCoT 11).The total number of monomorphic amplicons was 26 varied from 1 (primer SCoT 6) to 7 (primer SCoT 11) with an average of 3.25 monomorphic bands per primer. However, the total number of polymorphic band was 34 varied from 3 (SCoT 8 and SCoT 11) to 6 ( SCoT 10) with an average of 4.25 polymorphic amplicons per primer. The number of unique fragments varied from 0 (SCoT 1, 2, 6,11 AND 12) to 1 after (ScoT 3, 8 and 10)with an average of 0.37 unique fragments per primer.

The primer SCoT1 generated 5 monomorphic fragments and 4 polymorphic fragments with $44.4 \%$ of polymorphism. The fragments with molecular sizes 1550, 730, 515 and $175 \mathrm{bp}$. were detected nearly with all cultivars except cultivars Indian and German. Five polymorphic fragments with $71.5 \%$ polymorphism and two monomorphic fragments were generated by primer SCoT2 which appeared with cultivars Azoricum and Indian. The fragments with molecular sizes 2110, 700, 480 and 400 bp. has been identified with all cultivars except Indian, thus it could be considered as a negative selective markers for Indian cultivar. The fragment with molecular size 890 bp. was appeared with most of cultivars and absent in Holland and Indian cultivars. Four monomorphic fragments were exhibited at molecular sizes 1360, 485, 370 and 295 bp. and three polymorphic fragments and one positive marker (unique band) with molecular size 765 bp. was identified only with El alamy by primer SCoT3. On the contrary the fragments with molecular sizes 330 and 245 bp. were showed with most of the concerned cultivars and absent only with the German so, it can be considered as a negative marker for the German.

The highest polymorphism (85.7\%)

was observed by primer SCoT6. The fragment at molecular size 640 bp. was scored with cultivar Holland and Azoricum only. Negative markers have been detected with Indian by the fragments with molecular sizes $480,400,365$, 345 and 230 bp. SCoT8 primer scored $75 \%$ of polymorphism, two monomorphic fragments with molecular sizes 405 and 315 bp., three polymorphic fragment and another positive marker with molecular size 540 bp. was distinguished with Balady only. The fragment with molecular size 470 bp. was observed with all cultivars except cultivars Holland and Azoricum. Likewise the primer SCoT10 scored $66.6 \%$ polymorphism by three monomorphic fragments and six polymorphic fragment and generated one positive marker (unique band) at molecular size 645 bp. with cultivar Holland. The fragments with molecular sizes 1065, 1000, 785 and 655 bp. were presented with cultivars Holland and Azoricum only. The fragment at molecular size 500 bp. was scored with cultivars Holand, Azoricum and Elalamy. However the fragments with molecular sizes 415, 385 and 320 bp. were monomorphic. Primer SoT11 scored the lowest percentage of polymorphism 30\%, seven monomorphic fragments with molecular sizes 1535, 1390, 955, 850, 620, 510 and 435 bp. While the fragments at molecular sizes 305 and 230 bp. were identified with most of ecotypes except Balady and Elalamy. In a related the fragment at molecular size 300 bp. was recognized with all cultivars except cultivar German only, which in turn was considered as a negative marker for German. Primer SCoT12 scored two monomorphic fragments with molecular sizes 500 and 400 bp. and four poly morphic fragment with 
Walaa A. Ramadan et al.

$80 \%$ of polymorphism, the fragments at molecular sizes, 720, 680 and 450 bp. were detected with cultivars Azoricum and Elalamy and were absent with the other cultivars. In a related the fragment at molecular size 300 bp. was showed with cultivars Holland, Azoricum and Elalamy.

Finally three positive markers (3 unique bands) were generated by primers SCoT3, SCoT8 and SCoT10; the first one was appeared with cultivar El alamy, the second was identified with cultivars Balady and the third was observed with cultivar Holland.

Upon the obtained results of SCoT marker, the cluster analysis based on unweighted paired group method using arithmetic mean (UPGMA) were carried on and pooled together to generate the dendrogram and similarity index (Fig. 4 and Table 7).

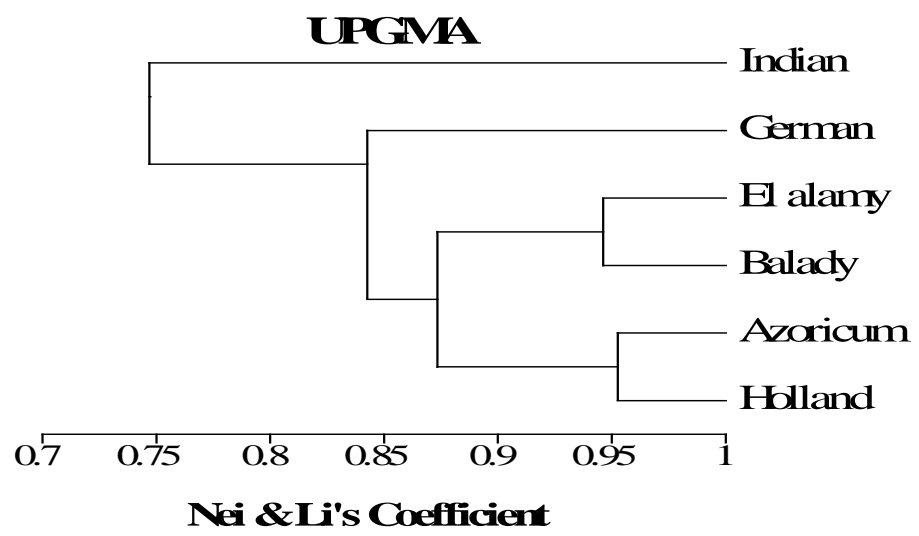

Fig. 4: Dendrogram represents the genetic relationships among the six fennel cultivars using UPGMA cluster analysis generated from of SCoT marker.

Table (7): Similarity index using SCoT analysis for the six fennel cultivars (Foeniculum Vulgare Mill.).

\begin{tabular}{|l|l|l|l|l|l|l|}
\hline Cultivars & Holland & Azoricum & Indian & German & Balady & El alamy \\
\hline Holland & 1 & & & & & \\
\hline Azoricum & 0.97 & 1 & & & & \\
\hline Indian & 0.72 & 0.71 & 1 & & & \\
\hline German & 0.82 & 0.83 & 0.79 & 1 & & \\
\hline Balady & 0.87 & 0.88 & 0.75 & 0.875 & 1 & \\
\hline El alamy & 0.86 & 0.89 & 0.74 & 0.86 & 0.97 & 1 \\
\hline
\end{tabular}

3- Combined analysis using ISSR and SCoT markers:

Regarding the collective data of ISSR and SCoT markers showed in table (8), the ISSR marker is more discriminating, provides more informative data than SCoT marker and can be reliable to evaluate the genetic diversity among the six examined cultivars as it performs the highest percentage of polymorphism $61.3 \%$ and higher unique bands number (4) which may define genetic finger printing that may be associated with one or more of the morphological traits (El-Atroush et al., 2014). 
Table (8): Comparison of genetic parameter between ISSR and ScoT analysis for the six fennel cultivars (Foeniculum Vulgare Mill.).

\begin{tabular}{|l|c|c|}
\hline \multirow{2}{*}{ Molecular parameter } & \multicolumn{2}{|c|}{ value } \\
\cline { 2 - 3 } & ISSR & SCoT \\
\hline Total bands & 38 & 59 \\
\hline Monomorphic bands & 15 & 26 \\
\hline Polymorphic bands & 23 & 34 \\
\hline \% of polymorphism & 60.3 & 57.6 \\
\hline Unique bands & 4 & 3 \\
\hline
\end{tabular}

The genetic similarity coefficient were gathered between the six fennel cultivars after the ISSR and SCoT markers as illustrated inTtable (9) and showed that the Azoricum cultivar is close distant to the
Holland (0.926) , Elalamy is close distant to Balady (0.922). This indicates that the relative genetic diversity of the cultivars is similar when SCOT and ISSR markers are used.

Table (9): Combined genetic Similarity index using ISSR and SCoT analysis for the six fennel cultivars (Foeniculum Vulgare Mill.).

\begin{tabular}{|c|c|c|c|c|c|c|}
\hline Cultivars & Holland & Azoricum & Indian & German & Balady & El alamy \\
\hline Holland & $\mathbf{1}$ & & & & & \\
\hline Azoricum & $\mathbf{0 . 9 2 6}$ & $\mathbf{1}$ & & & & \\
\hline Indian & $\mathbf{0 . 7 7 6}$ & $\mathbf{0 . 7 4 1}$ & $\mathbf{1}$ & & & \\
\hline German & $\mathbf{0 . 8 0}$ & $\mathbf{0 . 7 9 5}$ & $\mathbf{0 . 8 5 5}$ & $\mathbf{1}$ & & \\
\hline Balady & $\mathbf{0 . 8 3 6}$ & $\mathbf{0 . 8 3}$ & $\mathbf{0 . 7 4 2}$ & $\mathbf{0 . 8 1 1}$ & $\mathbf{1}$ & \\
\hline El alamy & $\mathbf{0 . 8 3 9}$ & $\mathbf{0 . 8 7 5}$ & $\mathbf{0 . 7 6}$ & $\mathbf{0 . 8 0}$ & $\mathbf{0 . 9 2 2}$ & 1 \\
\hline
\end{tabular}

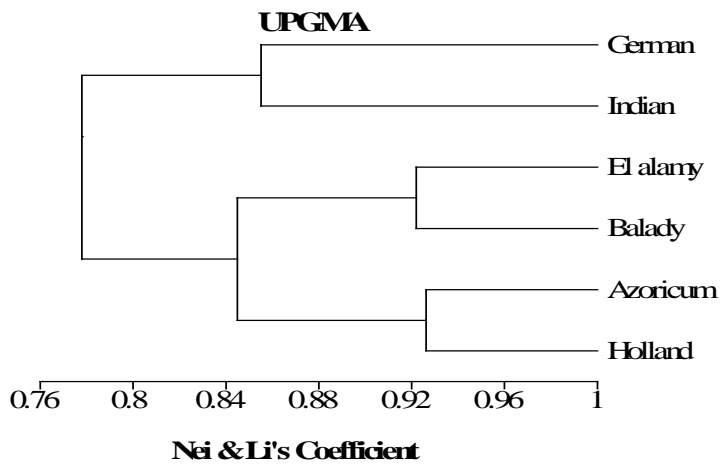

Fig. 5: Dendrogram represents the combined genetic similarity relationships among the six fennel cultivars using UPGMA cluster analysis generated from ISSR and SCoT markers. ABBREVIATIONS LIST

\begin{tabular}{|l|l|}
\hline CBDP & CAAT box derived polymorphism \\
\hline CDDP & Conserved DNA derived polymorphism \\
\hline ISSR & inter - simple sequence repeats polymorphism marker \\
\hline RAPD & Random amplified polymorphic DNA \\
\hline RFLP & Restriction fragment length polymorphism \\
\hline SCoT & Start codon targeted polymorphism marker \\
\hline SRAP & Sequence related amplified polymorphism \\
\hline SSR & Simple sequence repeats marker \\
\hline
\end{tabular}


Walaa A. Ramadan et al.

\section{Discussion}

Upon the results of ISSR analysis, the six studied cultivars were divided into two main groups with genetic diversity distance 0.76. The first group had the cultivars Balady and Elalamy, the second group comprises cultivars Holland, Azoricum, Indian and German. The second group was divided into two subgroups, the first one includes cultivars Indian and German, the second subgroup includes cultivars Holland and Azoricum. According to Nie,s and li,s coefficient the Genetic similarities coefficient between the cultivars ranged between 0.71 between Balady and Indian and between Balady and German to 0.92 as between Indian and German (Table 5). According to ISSR molecular marker German cultivar and Indian cultivar are close distant can exchange gene in between them smoothly despite the ultimate change in the environmental and weather conditions. Abou El-Nasr (2013) assessed the genetic relationships among three fennel cultivars (Balady, indian and Holland) by using 5 ISSR primers, and found that; the nearest relationship was detected between Indian and Holland varieties (77.00\%), followed by between (Balady and Holland) and (Balady and Indian), respectively.

In this respect, ISSR technique have been efficiently used for investigation of genetic diversity for different crops and medicinal plant species such as Phaseolus vulgaris L. (Galvan et al., 2003), Artemisia capillaries (Shafie et al., 2009), Lippia alba Mill. (Manica-Cattani et al., 2009) and Achillea millefolium (Farajpour et al., 2012). Bahmani et al. (2012) examined 25 ecotypes of F. vulgare Mill., collected from all over Iran using seven ISSR primers and had fifty two amplified fragments were generated 49 were polymorphic (91\% polymorphism). Shojaiefar et al. (2015) used 8 ISSR primers to investigate the variations in 18 fennel populations collected from Iran different regions and 2 European accessions. Mostafa and Abou Alhamd (2015) used 6 ISSR primers to identification salt tolerant mutants of $F$. vulgare which induced by dimethyl sulphate (DMS). These primers were generated 48 amplified bands, 45 of them were polymorphic (91.65\% polymorphism). Omid and Kalantar (2016) investigate over 30 ecotype of $F$. vulgare Mill in Iran by using different markers such as RAPD, ISSR, AFLP, RFLP and SRAP. The results have shown that, $36 \%$ polymorphic content (PIC). Zanganeh et al. (2016) used ISSR marker in order to study the genetic variation of 17 Iranian fennel ecotypes. A total of 51 DNA fragments were generated by ISSR markers, of which 45 were polymorphic (88.23\% polmorphism). Chitrangda and Malik (2018) assessed the genetic diversity for 14 fennel ecotypes by using molecular markers (ISSR, SCoT, CDDP and CBDP). Choudhary (2018) used two molecular marker techniques; RAPD and ISSR were used to evaluate the molecular diversity between 17 fennel cultivars. Ten ISSR primers were generated 59 fragments across 17 cultivars, of which 51 bands were polymorphic; $86.44 \%$ polymorphism.

On the other hand, SCoT marker was recently designed by Collard and Mackill (2009), then after it was employed as a new molecular marker technique to distinguish the genetic diversity and relationships between closely related cultivars. Miro et al. (2017) created twelve SCoT primers in assessment of fourteen Hungarian and international grape varieties and recommended one primer producing 17 polymorphic bands after data analysis, which was enough to distinguish the varieties. Mahjbi et al. (2015) used 12 SCoT primers to reveal polymorphism in Citrus genus. In potato, Gorji et al. (2011) 


\section{Assessment of genetic diversity among some fennel cultivars (Foeniculum vulgare Mill.) by} ISSR and SCoT Markers

employed 12 SCoT primers with 15 combinations generated 144 fragments. Xiong et al. (2011) depended on SCoT molecular marker polymorphism technique to study genetic diversity among 20 accessions of peanut. Moreover, Luo et al. (2010) used two molecular marker systems (SCoT and ISSR) in order to identify the genetic relatedness among 23 of mango germplasms.

SCoT analysis revealed that; the studied six cultivars have an average of genetic diversity distance 0.74 . They were divided into two main groups. The first group had the cultivar Indian only and the second comprises cultivars Holland, Azoricum, German, Balady and Elalamy. The second group was divided into two clusters at genetic diversity distance 0.83 ; the first one includes cultivar German only and the second include Elalamy, Balady, Azoricum and Holland at genetic diversity distance 0.87 then each cluster divided into two sub-clusters. The first subcluster comprises cultivars Balady, Elalamy and the second subcluster had cultivars Holland and Azoricum. According to Nie,s and li,s coefficient the Genetic similarities coefficient between the cultivars ranged between 0.71 as between Indian and Azoricum to 0.97 as between Elalamy and Balady and as between Azoricum and Holland ( Table 8). Worth to mention that the genetic similarties coefficient between Balady and Holland cultivars reaches 0.86 which may explain the success of planting Holland cultivar in Egypt despite the high difference in the environmental and weather conditions between Egypt and Holland.

As shown in Figure (5) the generated dendrogram from the combined results of ISSR and SCoT markers after the cluster analysis; resembles somehow the dendrogram generated after the ISSR marker. Kumar and Agrawal (2019) obtained the similar conclusion on the superiority of the ISSR marker (95.96\% polymorphism) over the SCoT marker (92.20\% polymorphism) when analyze genetic diversity and population structure among 52 Trichosanthes dioica Roxb. Previously the ISSR marker have been approved to be the more discriminating marker for the genetic diversity between Hordeum vulgre cultivars and landraces compared with RAPD and SSR Osman (2016). Jabbarzadeh et al. (2010) also found that the ISSR marker could provide more informative data than AFLP for genetic diversity analysis of rose species.

\section{CONCLUSION}

The ISSR and SCoT DNA based markers obtained data reveled that the examined six fennel cultivars were correlated and shared in their genetic pools and did not correspond to the geographical distribution origin. Among the two used molecular markers the ISSR marker is more discriminating, provides more informative data than SCoT marker.

\section{REFRENCES}

Abou El-Nasr, T.H.S.; Ibrahim, M.M.; Aboud, K.A. and Al-Kordy, M.A.A. (2013). Genetic variation among three fennel (Foeniculum vulgare Mill.) varieties on the basis of morphological characters, essential oil composition and ISSR markers. J. Appl. Sci. Res., 9(3): 1594-1603.

Bahmani, K.; Izadi-Darbandi, A.; Jafari, A.A.; Sadat, N.S.A. and Farajpour, M. (2012). Assessment of Genetic Diversity in Iranian Fennels Using ISSR Markers. J. Agric. Sci., 4(9): 79-84. doi.org/10.5539/jas.v4n9p79.

Chitrangda Y, Malik CP (2018). Genetic Variation among Fennel (Foeniculum vulgare Mill.) Varieties 
Walaa A. Ramadan et al.

on the basis of Essential Oil Composition and Molecular Markers (ISSR, SCoT, CDDP and CBDP). International Journal of Life Sciences 7(3):126-131.

Choudhary, S.; Sharma, R.; Meena, R.S. and Verma, A.K. (2018). Molecular diversity analysis in Fennel (Foeniculum vulgare Mill) Cultivars and its implications for conservation and Crop Breeding. Int. J. Curr. Microbiol. App. Sci., 7(3):794-809. doi.org/10.20546/ijcmas.2018.703.0 93.

Collard, B.C.Y. and Mackill, D.J. (2009). Start Codon Targted (SCoT) polymorphism: A simple novel DNA marker technique for generating gene -taegeted markers in plants. Plant Mol. Bio., 27:86-93.

Dellaporta, S.L.; Wood, J. and Hicks, J.B. (1983). A plant DNA preparation version II, Plant Mol. Biol. Rep., 4: 19- 21.

El-Atroush, H.; Barakat, H.M.; Tantawy, M.A. and El-Shabasy, A.E. (2014). Molecular characterization of Nitraria retusa and selected taxa of zygophyllaceae using RAPD and ISSR markers. Egypt. Genet. Cytology, (in press).

Farajpour, M.; Ebrahimi, M.; Amiri, R.; Golzari, R. and Sanjari, S. (2012). Assessment of genetic diversity in Achillea millefolium accessions from Iran using ISSR marker. Biochem. Syst. Ecol., 43: 73-79.

Galvan, M.Z.; Bornet, B.; Balatti, P.A. and Branchard, M. (2003). Inter simple sequence repeat (ISSR) markers as a tool for the assessment of both genetic diversity and gene pool origin in common bean (Phaseolus vulgaris L.). Euphytica, 132: 297301.
Gorji, A.M.; Poczai, P.; Polgar, Z, and Taller, J, (2011). Efficiency of arbitrarily amplified dominant markers (SCoT, ISSR and RAPD) for diagnostic fingerprinting intetraploid potato. Am. J. Potato Res., 88: 226-237.

Jabbarzadeh, Z.; Khosh-Khui, M.; Salehi, H. and Saberivand, A. (2010). Inter simple sequence repeat (ISSR) markers as reproducible and specific tools for genetic diversity analysis of rose species. Afr. J. Biotechnol., 9(37): 6091-6095.

Kumar, J. and Agrawal, V. (2019). Assessment of genetic diversity, population structure and sex identification in dioecious crop, Trichosanthes dioica employing ISSR, SCoT and SRAP markers. Heliyon, 5(3):e01346.

Lucinewton, S.; Raul, N.; Carvalho, J.; Mirian, B.; Lin, C. and Angela, A. (2005). Supercritical fluid extraction from fennel, (Foeniculum vulgare), global yield, composition and kinetic data. J. Supercritical Fluid, 35: 212219.

Luo, C.; He, X.H.; Chen, H.; Ou, S.J. and Gao, M.P. (2010). Analysis of diversity and relationships among mango cultivars using Start Codon Targeted (SCoT) markers. Biochem. Syst. Ecol., 38:1176-1184.

Mahjbi, A.; Ghada, B.; Amel, O. and Amel, S.H. (2015). Start Codon Targeted (SCoT) markers provide new insights into the genetic diversity analysis and characterization of Tunisian Citrus species. Biochem. Syst. Ecol., 61: 390-398.

Manica-Cattani, M.F.; Zacaria, J.G.; Pauletti, L.; Atti-Serafini, A. and Echeverrigaray, S. (2009). Genetic variation among South Brazilian accessions of Lippia alba Mill. 

ISSR and SCoT Markers

(Verbenaceae) detected by ISSR and RAPD markers. Braz. J. Biol., 69: 375-380.

Miró, K.; Nagy, T.; Korom, E. and Marincs, F. (2017). Discrimination of grape varieties by Start Codon Targeted genotyping using partially degenerate primers. Acta Biologica Szegediensis, 61(1):77-83.

Mostafa, G.G. and Abou Alhamd, F.M. (2015). Induction of salt tolerant mutants of Foeniculum vulgare by dimethyl sulphate and their identification using protein pattern and ISSR markers. Alex. J. Agric. Res., 60(2): 95-109..

Nan, P.; Peng, S.; Shi, S.; Ren, H.; Yang, J. and Zhong, Y. (2003). Interpopulation congruence in Chinese Primula ovalifolia reveled by chemical and molecular markers using essential oils and ISSRs. Zeitschrift für Naturforschung. J. Biosciences, 58: 57-61.

Nei, M. and Li, W.H. (1979). Mathematical model for studying genetic variations in terms of restriction endonucleases. Proc. Nat. Acad. Sci., 76: 52695273.

Omid, J. and Kalantar, M. (2016). Investigating Genetic Diversity of Foeniculum vulgare Mill using Molecular Markers. IJBR, 7(3): 109111.

Osman, S.A. (2016). Genetic studies on some Hordeum vulgare varities. PhD. Thesis, Ain shams University, Faculty of science.

Rather, M.A.; Bilal, A.D.; Shahnawaz, N.S.; Bilal, A.B. and Mushtaq, A.Q. (2016). Foeniculum vulgare A comprehensive review of its traditional use, phytochemistry, pharmacology and safety. Arabian J. Chem., 9(2): 1574-1583.
Sangwan, R.S.; Sangwan, N.S.; Jain, D.C.; Kumar, S. and Ranade, A.S. (1999). RAPD profile based genetic characterization of chemotypic variants of Artemisia annua L. Biochem. Mol. Int., 47: 935-944.

Shafie, M.B.; Sayed, S.M.; Hasan, Z. and Shah, R.M. (2009). Study of genetic variability of Worm wood capillary (Artemisia capillaries) using inter simple sequence repeat (ISSR) in Pahang region, Malaysia. Plant Omics J., 2: 127-13.

Shojaiefar, S.; Mirlohi, A.; Sabzalian, M.R. and Yaghini, H. (2015). Seed yield and essential oil content of fennel influenced by genetic variation and cultivar×year interaction. Industrial Crops and Products, 71: 97-105.

Wesam, K.; Maryam, M.; Sara, A.A.; Naim, S.; Majid. A.S. and Damoon, A.L. (2015). Therapeutic and pharmacological potential of Foeniculum vulgare Mill: a review. J. Herb. Med. Pharmacol., 2015; 4(1): 1-9.

Xiong, F.; Rubichun, Z.; Zhuqiang, H.; Jing, J.; Liangqiong, H.; weijian, Z. and Ronghua, T. (2011). Start Codon targeted polymorphism for evaluation of functional genetic variation and relationships in cultivated peanut (Arachis hypogaea L.) cultivars. Mol. Rep., 38:34873494.

Yadav, C. and Malik, C.P. (2016). Molecular Characterization of Fennel (Foeniculum vulgare Mill.) accessions using Start Codon Targeted (SCoT) markers. J. Plant Sci. Res., 32 (1): 37.

Yong-Cui, H.; Ze-Hong, Y.; Yu-Ming, W. and You-Liang, Z. (2005). Genetic diversity in barley from west China based on RAPD and ISSR analysis. 
Walaa A. Ramadan et al.

Barley Genetics Newsletter, 35: 922.

Zanganeh, K.; Fakheri, B.; Orooji, F.; Afzalifar, A. and Makhdoomi, M.A. (2016). Study of genetic diversity in Fennel (Foeniculum vulgare) ecotypes using ISSR marker. J. Medicinal Plants Biotechnol., 2(4): 10-19.

Zietkiewicz, E.; Rafalski, A. and Labuda, D. (1994). Genome fingerprinting by simple Sequence Repeat (SSR): anchored polymerase chain reaction, Genomic, 20: 176-183.

تقييم التنوع الوراثي بين بعض أصناف الثمر Foeniculum vulgare Mill) بواسطة ISSR و SCoT Markers

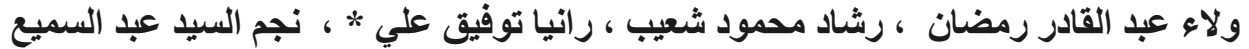

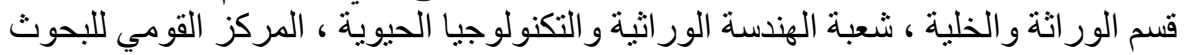

$$
\begin{aligned}
& \text { raniatawfick@hotmail.com البريد الالكترونى للباحث الرئيسئ }
\end{aligned}
$$

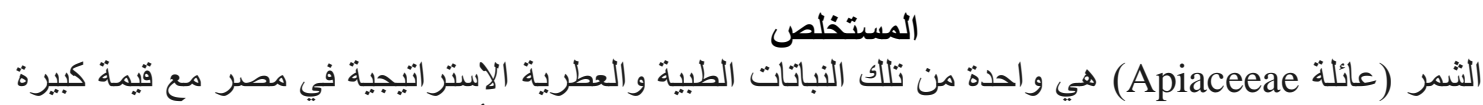

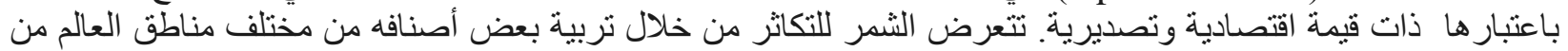

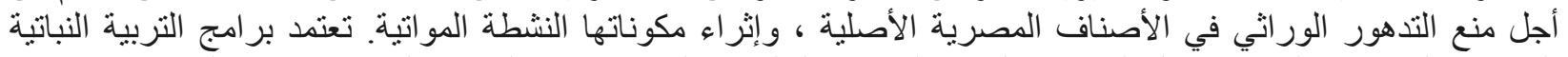

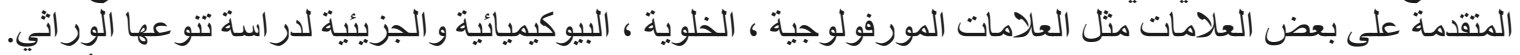

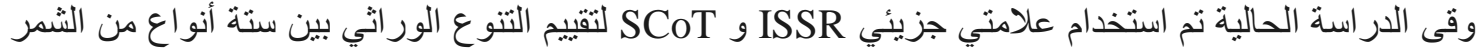

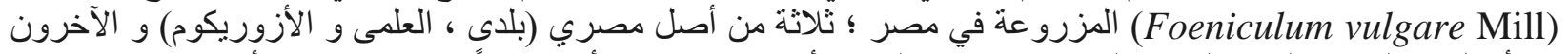

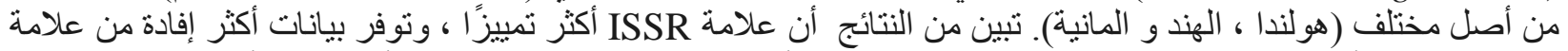

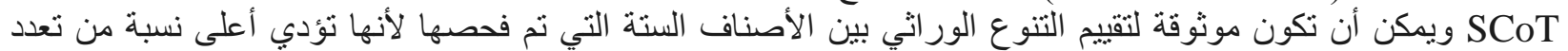

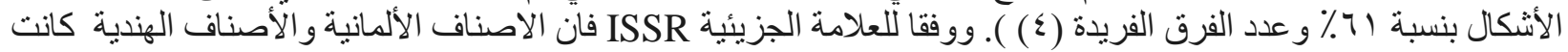

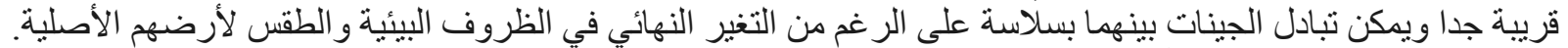

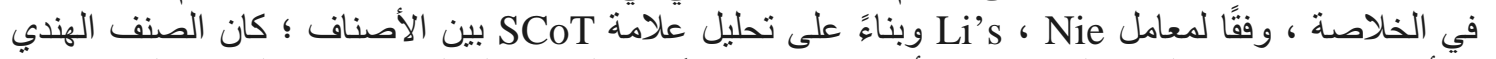

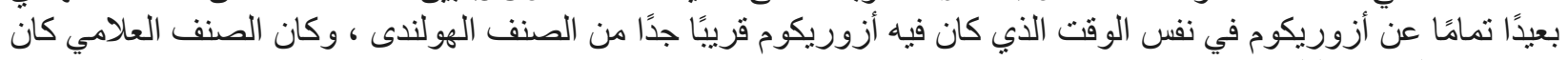

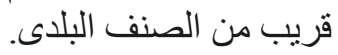

